\title{
The Treatment of Spatial Incoherence in the Aberration Corrected STEM
}

\author{
M. P. Oxley,*** and S. J. Pennycook**** \\ * Department of Physics and Astronomy, Vanderbilt University, Nashville, TN 37235 \\ ** Materials Science and Technology Division, Oak Ridge National Laboratory, PO Box 2008, Oak \\ Ridge, TN 37831
}

Aberration correction of the probe forming optics of the scanning transmission electron microscope (STEM) has resulted in images with higher contrast and increased signal to noise ratios. This provides the opportunity for truly quantitative microscopy and direct comparison of imaging theory and experiment. Recent work has suggested that the inclusion of spatial incoherence in simulations is essential to obtain quantitative agreement between theory and experiment [1]. In the past, computationally convenient approximations such as partial coherence have been adequate in many cases, but the larger probe forming aperture sizes in aberration corrected STEMs make such approximations poor. We will examine the affect of spatial incoherence on various imaging modes in the STEM.

Spatial incoherence, due to finite source size, may be modeled as a shift in the probe position. The probe intensity can then be constructed by adding the correctly weighted contributions from electrons originating from different positions on the source. Assuming the source size can be described by a Gaussian distribution, we can derive an envelope function describing spatial incoherence in the form

$$
E_{S}(\mathbf{g}, \mathbf{h})=\exp \left[-\pi^{2} S^{2}(\mathbf{g}-\mathbf{h})^{2}\right] .
$$

Here $\mathbf{g}$ and $\mathbf{h}$ are reciprocal space vectors and $S$ is the 1/e value of the shift in probe position. The probe intensity can then be calculated using

$$
I(\mathbf{r})=\iint \Psi(\mathbf{g}) \exp (2 \pi i \mathbf{g} \cdot \mathbf{r}) E(\mathbf{g}, \mathbf{h}) \Psi^{*}(\mathbf{h}) \exp (-2 \pi i \mathbf{h} \cdot \mathbf{r}) d \mathbf{g} d \mathbf{h}
$$

where $\Psi(\mathbf{g})$ is the reciprocal space wave function of the probe. Evaluation of Eq. (2) is computationally expensive, especially for large probe forming apertures where the range of integration is increased. A common approach is to assume partial coherence, i.e. $E_{S}(\mathbf{g}, \mathbf{h})=E_{S}(\mathbf{g})$ $E_{S}(\mathbf{h})$ where

$$
E_{S}(\mathbf{g})=\exp \left(-\pi^{2} S^{2} \mathbf{g}^{2}\right)
$$

Equation (2) may then be evaluated using an inverse fast Fourier transform. In Fig. 1 we examine the affect of increasing source size for a range of probe forming apertures. All calculations assume an aberration free probe formed with $100 \mathrm{keV}$ incident electrons. Results using the full spatial incoherence envelope function are shown in red, while the simulations using partial coherence are shown in blue. For a $10 \mathrm{mrad}$ probe forming aperture, typical of a machine without aberration correction, low to moderate levels of incoherence are reasonably described by partial coherence. But for parameters more typical of aberration corrected machines, partial coherence clearly underestimates the broadening of the probe. 
For the calculation of elastic and inelastic images, the envelope functions may be included in a Bloch wave formulation [2]. In most cases the specimen itself has been ignored (or approximated as a phase object) in the treatment of incoherence. Here the envelopes act directly on the Bloch states, leading to the possibility of different levels of damping for different materials. We will examine the effects of incoherence on various inelastic imaging modes.

References

[1] L.M. LeBeau et al., Phys. Rev. Lett. 100 (2008) 206101.

[2] L.J. Allen et al., Ultramicroscopy 96 (2003) 47.

[3] Research sponsored by the Office of Basic Energy Sciences, Division of Materials Sciences and Engineering.
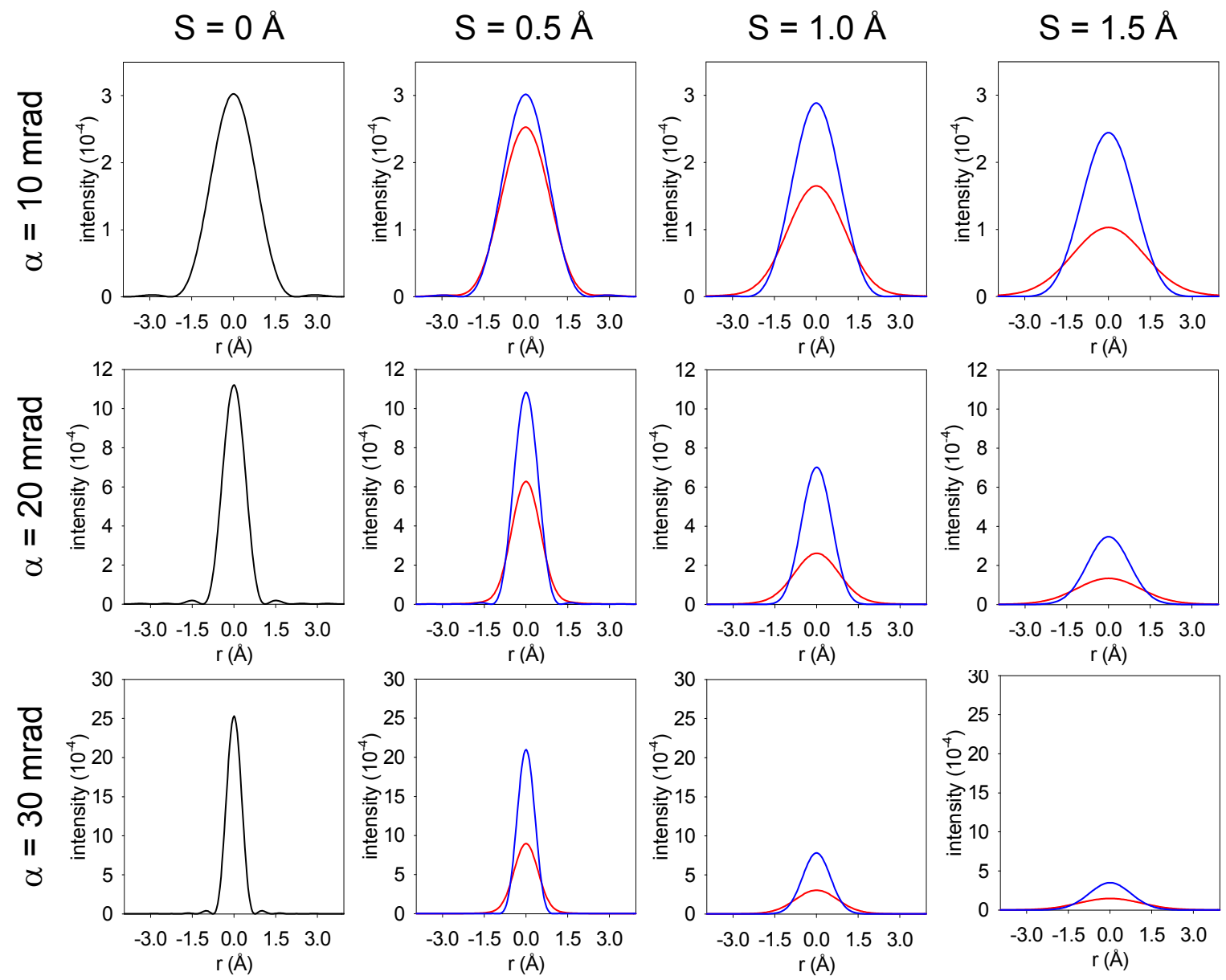

FIG. 1. Calculated STEM probe intensities for $100 \mathrm{kV}$ incident electrons as a function of probe forming aperture $\alpha$ and source size S. The blue lines represent the intensity calculated assuming partial coherence. The red lines show the intensity calculated using the full spatial coherence envelope function. 